\title{
La independencia del Banco Central y el desempeño macroeconómico: algunas evidencias comparativas
}

El grado de independencia del Banco Central varía considerablemente entre los distintos países. Varios autores, entre ellos Bade y Parkin (1982), Alesina (1988, 1989) y Grilli, Masciandaro y Tabellini (1991,) encontraron que los bancos centrales más independientes están asociados con niveles más bajos de inflación. En esta nota se investiga si se puede encontrar un correlación entre la independencia del Banco Central y el nivel y la variabilidad de las variables económicas reales, tales como el crecimiento, el desempleo y tasas de interés real. Nuestra conclusión es que si bien la independencia del Banco Central promueve la estabilidad de los precios, no tiene ningún impacto mesurable sobre desempeño económico real.

\section{Teoría}

Como señala Rogoff (1985), las teorías de inconsistencia dinámica de la inflación del tipo de las desarrolladas en Kydlan y Prescolt (1977) y Barro y Gordon (1983), hacen plausible que los bancos centrales más independientes reduzcan la tasa de inflación. La delegación de la política monetaria a agentes cuyas preferencias son menos favorables a la inflación que las preferencias de la sociedad, sirve como un mecanismo de compromiso que permite mantener un nivel más bajo de inflación de lo que sería posible de otra manera. Alesina y Grilli (1992) desarrollan este argumento y demuestran que el "votanle medio" quería tener un Banco Central menos favorable a la inflación que él mismo. Sin embargo, el "votante medio" quiere ser "inconsistente en el tiempo" y retira al funcionario encargado del Banco Central, quien, ex post, está siendo demasiado conservador en maleria de inflación.

Al aislar la política monetaria del proceso político, se evita este problema y se ayuda a reforzar el equilibrio de baja inflación. Sin algún grado de independencia política sería imposible tener un Banco Central menos favorable a la inflación que la mayoría de los votantes, lo cual constituye una meta social deseable. 
¿Cuál es el efecto de la independencia del Banco Central sobre las variables reales? Si se mantiene el supuesto de que la política monetaria tiene efectos reales, los argumentos plausibles señalan en varias direcciones. La independencia del Banco Central podría mejorar el desempeño económico real por varias razones. En primer lugar, un Banco Central independiente que esté libre de presiones políticas podría comportarse más de una manera más predecible, promoviendo la estabilidad económica y reduciendo las primas de riesgo en las tasas de interés real. Más específicamente, un Banco Central independiente podría aislar a la economía de los ciclos económicos polílicos, ya sea previniendo la manipulación preelectoral de la política monetaria como en los modelos de Nordhaus (1975) y Rogoff y Sibert (1988), o reduciendo los shocks partidarios sobre las políticas aplicadas después de las elecciones, como en los modelos de Hibbs (1987) y Alesina $(1988,1989)$. Para un análisis teórico y empírico más amplio de la política monetaria y los ciclos económicos políticos, ver Willett (1988)².

En segundo lugar, en la medida en que la alta inflación tiene efectos adversos sobre el desempeño económico, ya sea creando dislorsiones, estimulando las aclividades en busca de rentas, o aumentando las premias de riesgo, se esperaría que la independencia del Banco Central mejorara el desempeño económico. Si, como a menudo se sugiere (por ejemplo, Romer y Romer, 1989), la mayoría de las recesiones de Estados Unidos se debe a que la Reserva Federal quebranta la inflación después de haberle permitido aumentar demasiado, podría esperarse que una política contraria más consistente a la inflación estaría asociada con un desempeño económico menos variable.

Por otra parte, los argumentos tradicionales en favor de las políticas monetarias que responden a razones políticas, sostienen que los bancos centrales políticamente sensibles quizá estén más preocupados que los bancos centrales independientes por el aumento del producto y la disminución del desempleo y de las tasas de interés real. Si la política monetaria pudiera lograr estos objetivos, podría esperarse que los bancos centrales independientes lograran tasas menores de inflación a costa de un desempeño económico real inferior. Rogoff (1985) proporciona un modelo formal de esta compensación; en su modelo, los bancos centrales más aversos a la inflación aplican políticas económicas de estabilización menos discrecionales y, por lo tanto, toleran una mayor variabilidad ciclíca en la actividad económica.

El impacto de la independencia del Banco Central sobre el desempeño económico constituye, en última instancia, una cuestión empírica. En consecuencia, veamos los datos pertinentes.

\section{Medición de la independencia del Banco Central}

Al examinar la independencia del Banco Central, la principal dificultad reside en medir su independencia en diferentes países, tarea que han encarado varios 
autores. Bade y Parkin (1982) construyeron una escala (del 1 al 4) de la dicha independencia para doce países, basada en la "independencia política" del Banco Central. Utilizando el mismo criterio de Bade y Parkin, Alesina (1988) agrega cuatro paises más. Se considera que la independencia política depende de la relación institucional entre el Banco Central y el poder ejecutivo; del procedimiento de nombrar y destituir al presidente del banco; del papel de los funcionarios del gobierno en el directorio de la institución, y de la frecuencia de los contaclos entre el poder ejecutivo y el banco. Nos basamos en la versión enmendada de la escala de Bade y Parkin contenida en Alesina (1988).

Más recientemente, Grilli, Masciandaro y Tabellini (1991) construyeron una medida relacionada con la independencia del Banco Central, que refleja tanto la "independencia política" como la "independencia económica". La independencia política se define, en esencia, como en Bade y Parkin (1982), como la capacidad del Banco Central para seleccionar sus objetivos políticos sin la influencia del gobiemo. Esta medida se basa en factores tales como si el gobemador y el directorio son nombrados por el gobiemo, la duración de sus nombramientos, si los representantes de gobierno participan en el directorio del banco, si se requiere que el gobiemo apruebe las decisiones de política monetaria, y si el objelivo es la "estabilidad de precios" explícila y prominentemente expresado en los estatutos del banco central. "La independencia económica" se define como la capacidad para utilizar instrumentos de política monetaria sin restricciones. La limilación más común impuesta sobre la conducción de la política monetaria, es la medida en que se requiere que el Banco Central financie el déficit gubernamental. Este índice de la independencia económica mide esencialmente cuán fácil es para el gobiemo financiar los déficits por medio del acceso direclo al crédito del Banco Central ${ }^{3}$.

El Cuadro 1 subraya los dos índices y nuestro procedimiento: ambos índices indican un aumento en la cantidad de independencia y son ampliamente consistentes entre sí $^{4}$. En el análisis empírico que sigue utilizamos el promedio de los dos índices, el cual se indica en la última columna del Cuadro 1. Los resultados fueron muy similares cuando se utilizó cualquiera de las dos escalas de manera individual ${ }^{5}$.

Debe señalarse que las clasificaciones resumidas anteriormente reflejan las leyes y constiluciones de los bancos centrales. Dichas leyes están sujetas a cambios, aunque muy esporádicos. Estas clasificaciones descritas son relevantes para el período de muestra estudiado aquí, 1955-88. Hasla hace poco, el grado de independencia del Banco Central ha aumentado en algunos países. Estos cambios son, sin embargo, demasiado recientes para ser relevantes para nuestra muestra ${ }^{\mathrm{A}}$. 


\section{Cuadro 1}

Indice de la independencia del Banco Central

\begin{tabular}{lrrcr}
\hline País & BP* & GMT* & $\begin{array}{c}\text { Conversión } \\
\text { de GMT a BP*. }\end{array}$ & $\begin{array}{r}\text { Promedio } \\
\text { GMT, BP*.. }\end{array}$ \\
\hline Australia & 1 & 9 & 3 & 2 \\
Bélgica & 2 & 7 & 2 & 2 \\
Canadá & 2 & 11 & 3 & 2.5 \\
Dinamarca & 2 & 8 & 3 & 2.5 \\
Francia & 2 & 7 & 2 & 2 \\
Alemania & 4 & 13 & 4 & 4 \\
Ilalia & 1.5 & 5 & 2 & 1.75 \\
Japón & 3 & 6 & 2 & 2.5 \\
Países Bajos & 2 & 10 & 3 & 2.5 \\
Noruega & 2 & NA & NA & 2 \\
Nueva Zelandia & 1 & 3 & 1 & 1 \\
España & 1 & 5 & 2 & 1.5 \\
Suecia & 2 & NA & NA & 2 \\
Suiza & 4 & 12 & 4 & 4 \\
Reino Unido & 2 & 6 & 2 & 2 \\
Estados Unidos & 3 & 12 & 4 & 3.5 \\
\hline
\end{tabular}

* Este es el indice originalmente propuesto por Bade y Parkin (1982) y extendido por Alesina (1988).

** Suma de los índices de la independencia económica y política calculados por Grilli, Manciandaro y Tabelli (1991).

*** Conversión de la escala GMT a una escala de 1 a 4 comparable con la escala BP. La conversión es la siguiente:

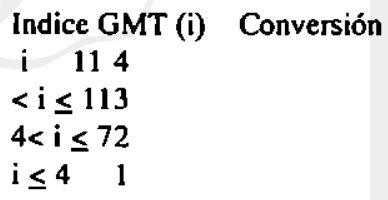

**** Promedio de las columnas (1) y (3).

\section{La independencia del Banco Central y el desempeño macroeconómico}

Nuestro procedimiento empírico es extremadamente sencillo. Trazamos varias medidas del desempeño económico que abarca todo el período 1955-1988, contra medidas de la independencia del Banco Central. Utilizando la información conlenida en el Apéndice (Cuadros A1 y A2), hemos verificado que se 
obtienen resultados similares para el shock posterior a la crisis petrolera de 19731978. El gráfico verifica lo que pusieron de relieve los trabajos previos: una correlación negativa casi perfecta entre la inflación y la independencia de Banco Central. Dada la correlación bien documentada que existe entre el nivel y la variabilidad de la inflación, no es sorprendente que el gráfico $1 \mathrm{~b}$ revele una fuerte relación negativa entre la variabilidad de la inflación y la independencia de Banco Central.

Las Gráficas $2 a$ y $2 b$ investigan las relaciones entre la independencia del Banco Central y el nivel o la variabilidad del crecimiento económico. No se observa relación alguna. Suiza, que ha tenido un Banco Central extremadamente independiente, muestra un crecimiento mucho más lento y variable que el país promedio en la muestra, mientras que Alemania y los Países Bajos, que también han tenido bancos centrales relativamente independientes, han mostrado un desempeño satisfactorio desde el punto de vista económico. Por otro lado, los países con bancos centrales relativamente dependientes, como España y Nueva Zelandia, han mostrado un crecimiento económico relativamente variable, mientras que Francia, con un Banco Central relativamente dependiente, ha gozado de un crecimiento constante ${ }^{7}$. Se obtienen resultados análogos si se utiliza el crecimiento del Producto Interno Bruto per cápita, como lo muestran las Gráficas $3 a$ y $3 b$.

\section{Gráfica $1 a$}

Inflación promedio

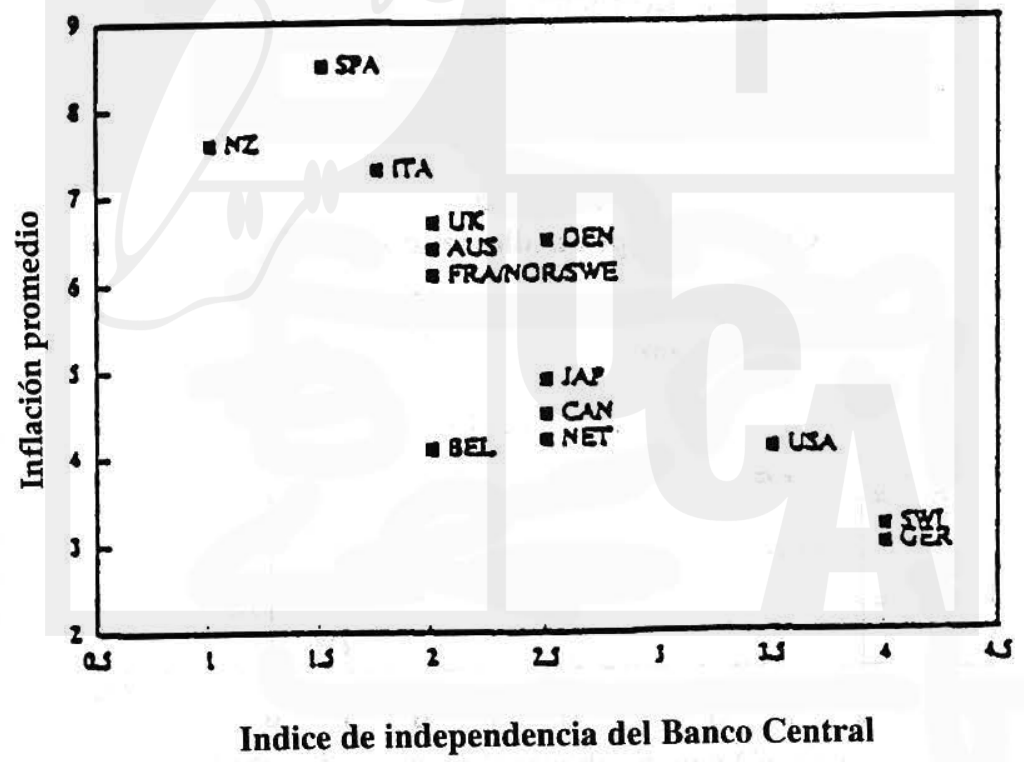


Las Gráficas $4 a$ y $4 b$ repiten el análisis para el desempleo. A pesar del hecho observado por Summer y Wadwhami (1989) de que la correlación entre el desempleo y el crecimiento del PNB real es bajo, las medidas del desempleo tampoco parecen estar relacionadas estrechamente con las medidas de la independencia del Banco Central.

Las Gráficas $5 a$ y $5 b$ analizan la relación que existe entre la independencia del Banco Central y las tasas de interés real. No se observa una relación clara entre la independencia y las tasas de interés real promedio ex post. Ni tampoco lo logra una comparación de la independencia del Banco Central con las medidas de tasa la de interés real ex ante (no mostradas) preparadas por Barro.

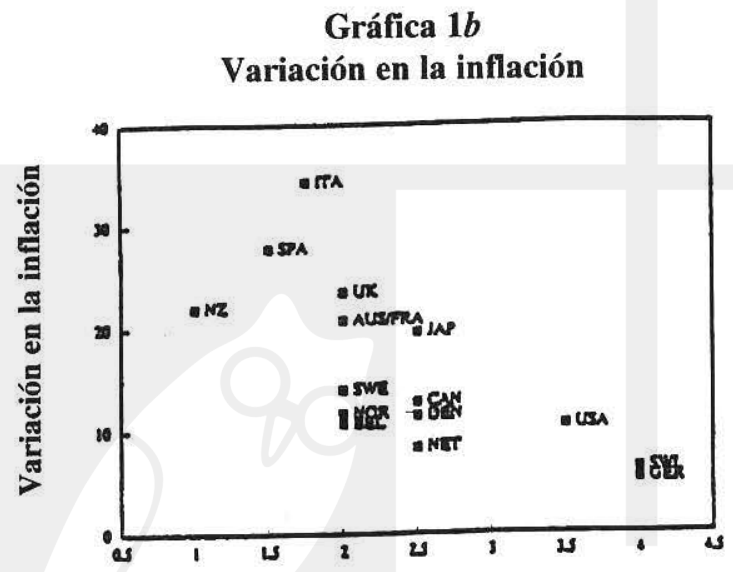

Indice de independencia del Banco Central

Gráfica $2 a$

Crecimiento promedio del PNB real

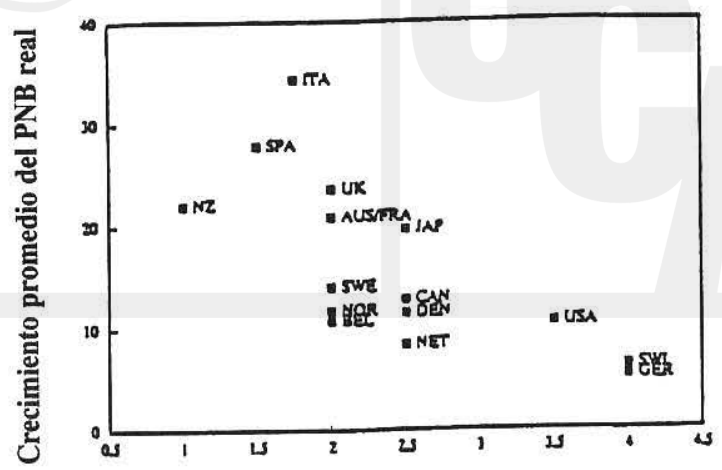

Indice de independencia del Banco Central 
Gráfica $2 b$

Variación del crecimiento PNB real

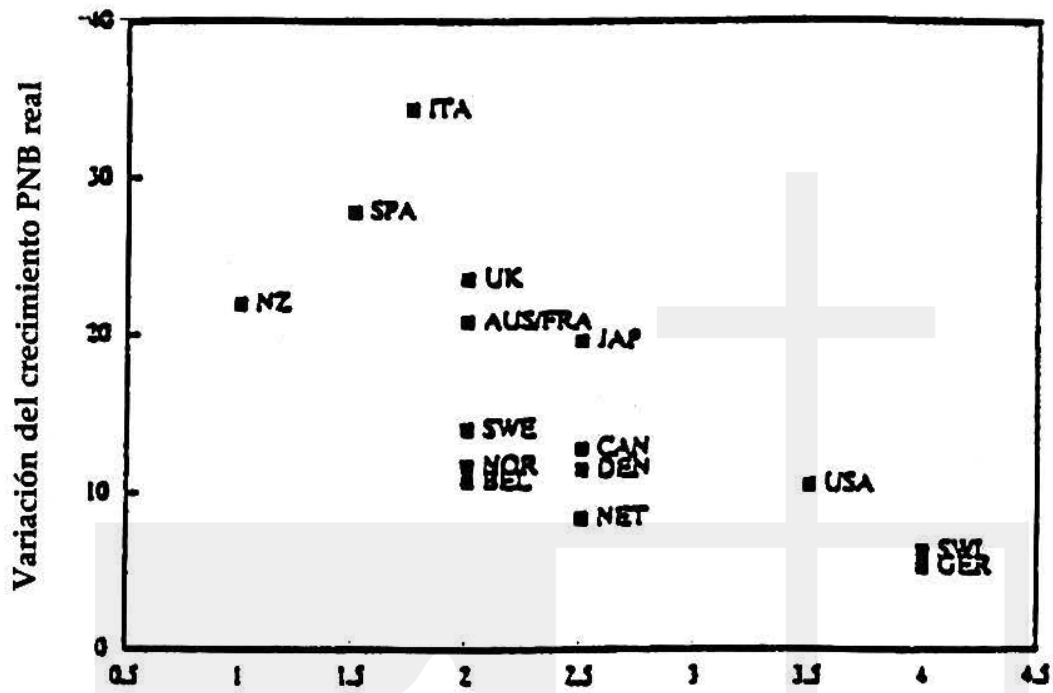

Indice de independencia del Banco Central

Gráfica 3a

Crecimiento promedio del PNB per cápita

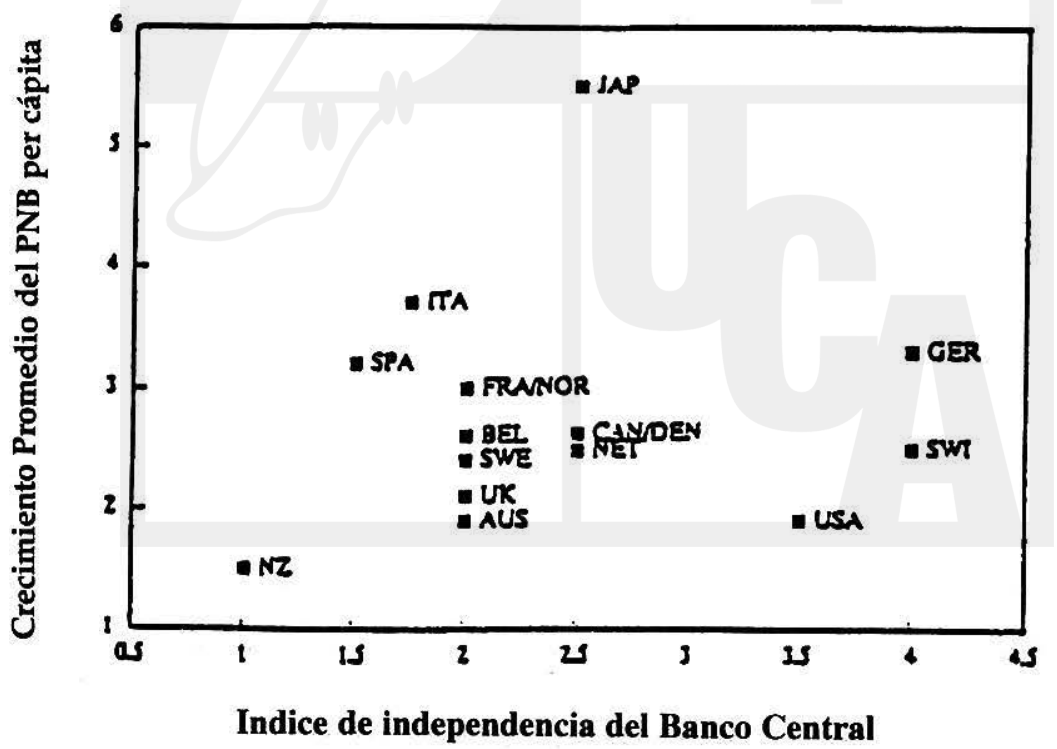



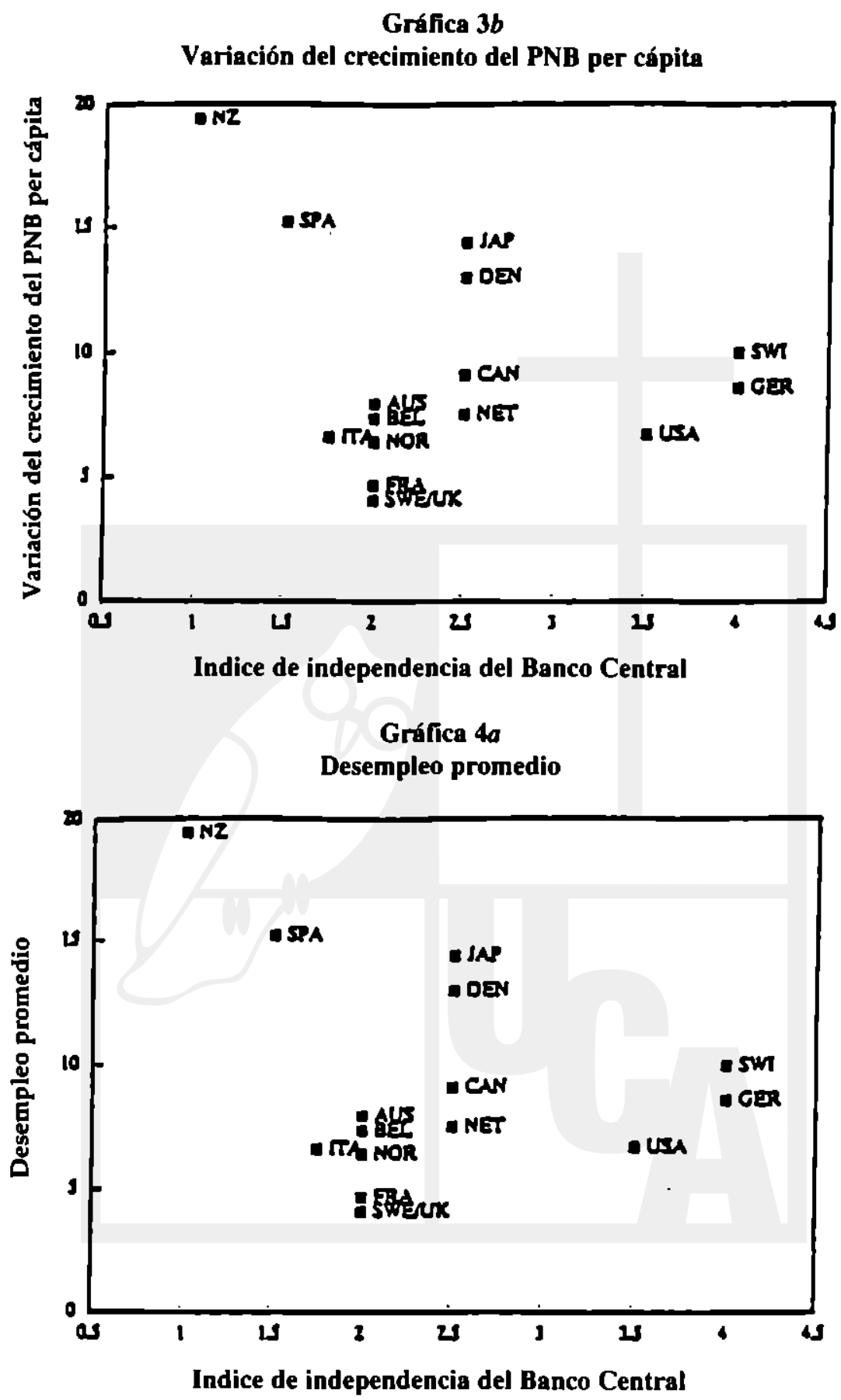
Gráfica $4 b$

Variación del desempleo

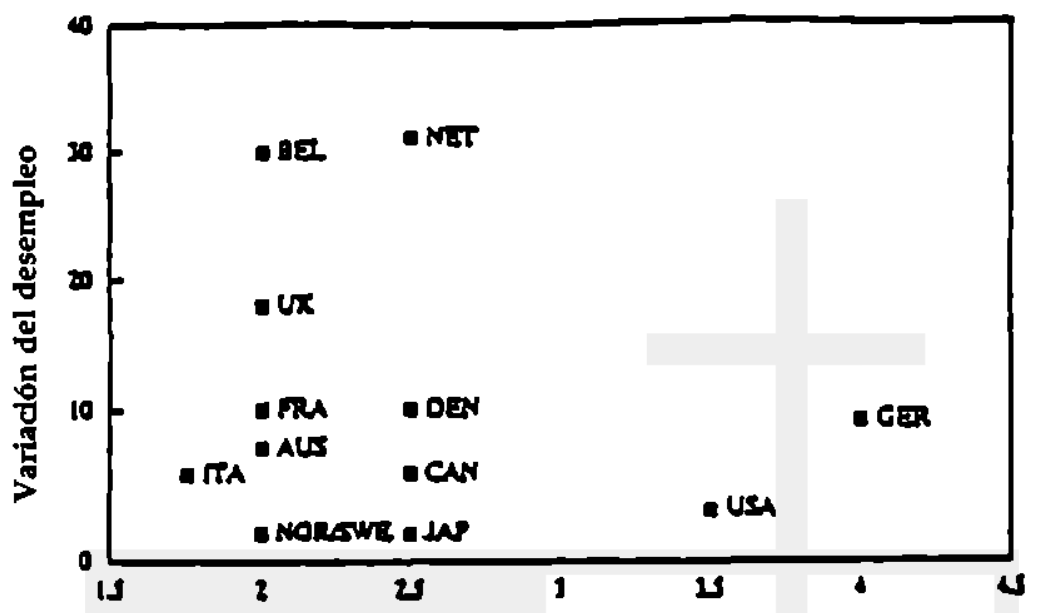

Indice de independencia del Banco Central

Gráfica $5 a$

Tasa promedio de interés real

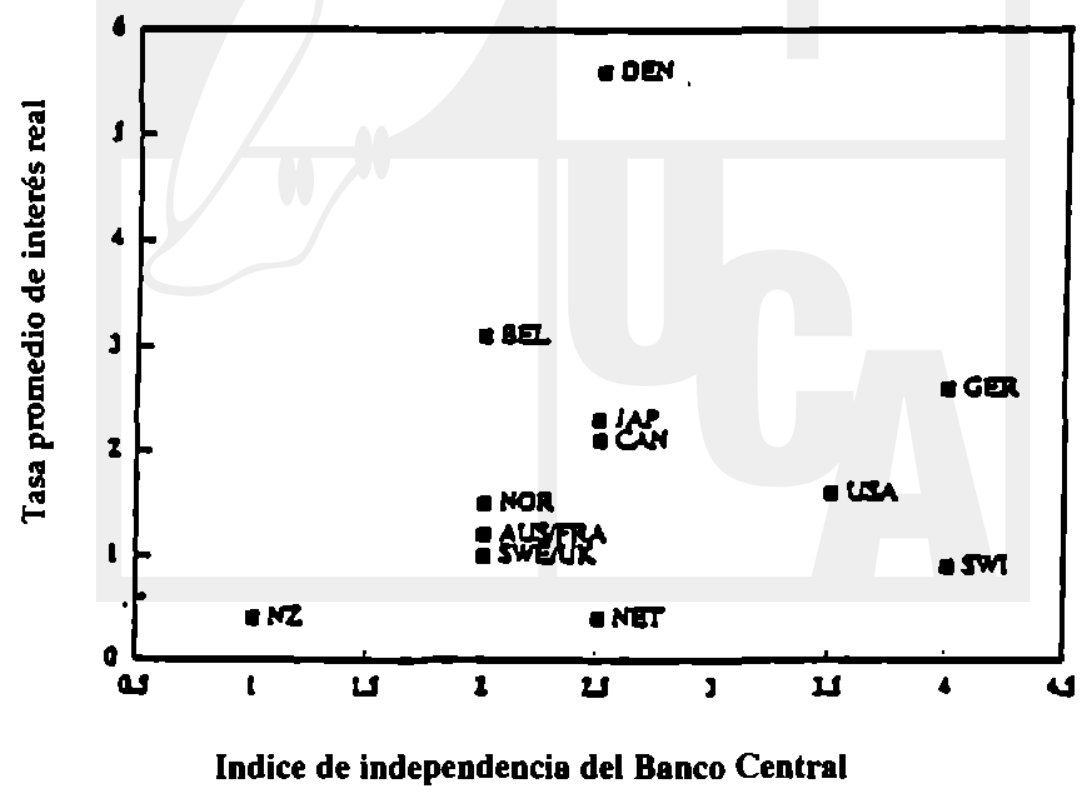

$R 660^{\circ}$ 
Sala-i Martin (1990), que revelan el mismo patrón. Si bien una política monetaria expansionista podría influenciar las tasas de interés real a corto plazo, no parece que una política monetaria sistemáticamente expansionista (por lo menos del tipo que proporcionan los bancos centrales dependientes) reduzca las tasas de interés real a lo largo de un período prolongado. Por otra parte, como podría esperarse dadas nuestras comprobaciones acerca de la variabilidad de la inflación, existe una relación claramente negativa entre la independencia del Banco Central y la variabilidad de las tasas de interés real ex post.

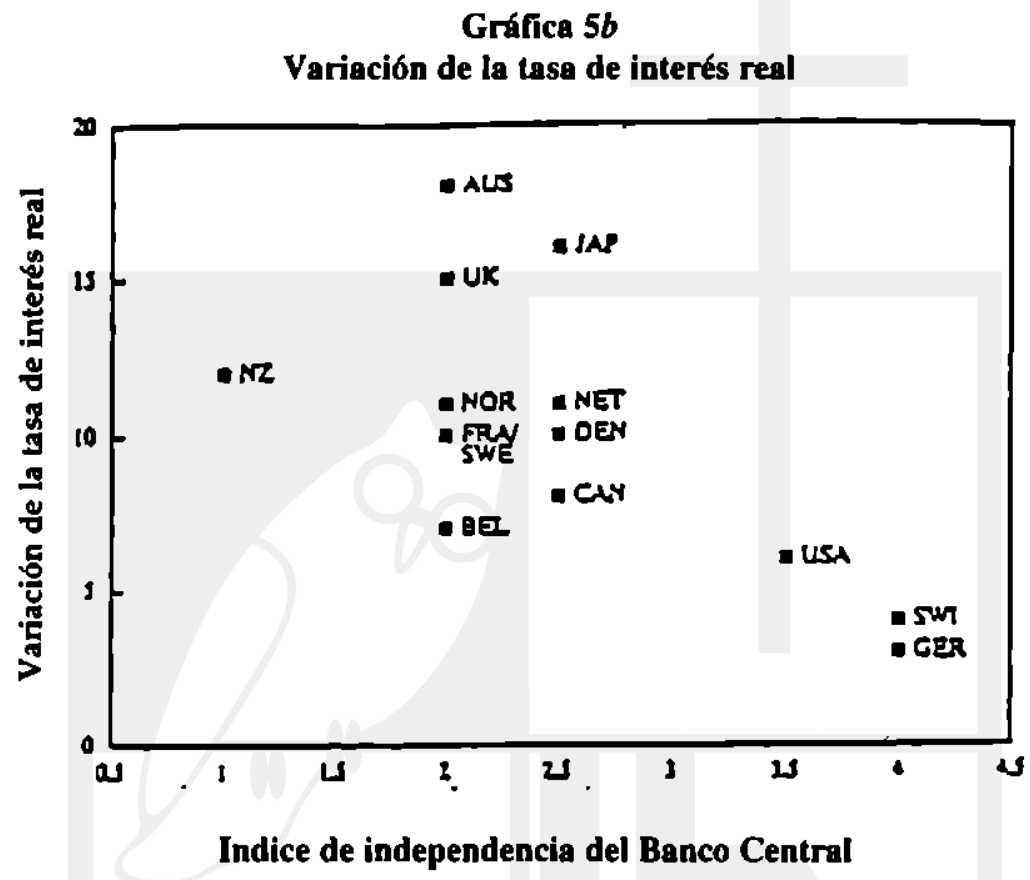

\section{Conclusiones}

Eslos resultados sugieren que la disciplina monetaria asociada a la independencia de Banco Central reduce el nivel y la variabilidad de inflación, pero no liene ni grandes beneficios o costos en términos del desempeño macroecómico real. Esta observación representa, por lo menos, un fragmento de evidencia en apoyo de las teorías que enfatizan la neutralidad del dinero.

Nuestras comprobaciones también tienen implicaciones para el debate que está manteniéndose sobre las reglas óptimas que rigen la política monetaria. Más obviamente sugieren que los resultados económicos ameritan la independencia del Banco Central. De manera más sutil, plantean dudas acerca de los beneficios 
de las políticas monetarias basadas en normas. Los defensores de estas políticas típicamente enfatizan que las mismas evitarían la inflación de consistencia dinámica. Las comprobaciones de este trabajo sugieren que es posible que las naciones logren estos beneficios, sin fijar una regla monetaria aislando al Banco Central del control político. Si bien es posible que el desempeño basados en reglas serían superiores al desempeño discrecional por motivos de estabilización, Summers (1988) presenta una serie de razones por las cuales ello resulta improbable, incluyendo acontecimientos no provistos y la posibilidad de que una economía quede atrapada alrededor de un equilibrio subóptimo en el cual la estabilización no sería deseable ${ }^{\sharp}$.

Los resultados no son concluyentes en el sentido de que sólo hemos observado los datos de una manera muy directa; se justifica un análisis más detallado de la relación que existe entre la independencia del Banco Central y los resultados reales. Por ejemplo, podría ser útil utilizar la independencia del Banco Central como un instrumento al estudiar los efectos de las diferencias en las políticas monetarias entre países, 0 incluir variables adicionales de control del tipo considerado en Summers y Wadwhani (1989), al evaluar el impacto de la independencia del Banco Central en los resultados económicos.

Además, el grado de independencia del Banco Central es sólo uno de los varios factores institucionales, arreglos cambiarios y shocks exógenos que influencian los resultados económicos en los diferentes países'. Nuesiros resultados, sin embargo, crean alguna presunción que los beneficios inflacionarios de la independencia del Banco Central probablemente van a ser superiores a los costos en materia de producto.

Por último, el grado de independencia del Banco Central puede ser una variable endógena. Por ejemplo, la experiencia histórica de una hiperinflación en Alemania, podría haber aumentado la aversión del público alemán contra la inflación y su propensión a tener un Banco Central independiente, comprometido en favor de la estabilidad de precios. Dentro del periodo de muestra considerado en este estudio, es un supuesto razonable considerar que las leyes de la banca central son constantes y exógenas. Un análisis más "histórico" sobre la evolución a largo plazo de los arreglos institucionales debería abordar temas relacionados con la endogeneidad conjunta entre los resultados económicos y las instituciones. 


\section{Cuadro A1 \\ Apéndice \\ Independencia del Banco Central y resultados económicos}

\begin{tabular}{|c|c|c|c|c|c|c|c|c|c|c|c|}
\hline Pais & $\begin{array}{l}\text { Indice frumedio } \\
\text { de independerein } \\
\text { del Banos Ceatril }\end{array}$ & $\begin{array}{l}\text { Inflacto } \\
\text { promadio } \\
1955-88\end{array}$ & $\begin{array}{l}\text { Variación } \\
\text { a b influción } \\
\text { lo55.88 }\end{array}$ & $\begin{array}{c}\text { Crecimiealo } \\
\text { promedio del } \\
\text { PI8 ral 1955-07 }\end{array}$ & $\begin{array}{l}\text { Variación en ell } \\
\text { crecimiemo del } \\
\text { P[B real 1955.87 }\end{array}$ & $\begin{array}{l}\text { Crecimiento } \\
\text { Proenedio del } \\
\text { PIB real por cipita } \\
1955-87\end{array}$ & 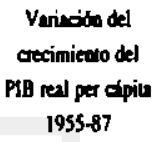 & $\begin{array}{c}\text { Tese promedio } \\
\text { de desempleo } \\
1958-88\end{array}$ & $\begin{array}{l}\text { Variación de } \\
\text { La vese de } \\
\text { dresempleo } \\
1958-88\end{array}$ & $\begin{array}{c}\text { Vuriación de } \\
\text { inierts real } \\
1957.80\end{array}$ & $\begin{array}{r}\text { Variación } \\
\text { del interts } \\
\text { real } 1957.80\end{array}$ \\
\hline Eqpis & 1.5 & as & 27.8 & 4.2 & 9.4 & 3.2 & 15.2 & N/A & N/A & N/A & N/A \\
\hline Nueva Zelandin & 1 & 7.6 & 21.9 & 3.0 & 5.4 & 15 & 19.4 & N/A & N/A & 0.4 & 12.0 \\
\hline Ausuralin & 20 & 6.4 & 20.8 & 4.0 & 4.6 & 1.9 & 7.9 & 4.7 & 7.0 & 1.1 & 18.0 \\
\hline Reino Unido & 2 & 67 & 235 & 24 & 4.0 & 21 & 4.4 & 5.3 & 18.0 & 1.0 & 15.0 \\
\hline Fnncis & 2 & 6.1 & 20.9 & 3.9 & 4.0 & 3.0 & 4.6 & 4.2 & 10.0 & 1.1 & 10.0 \\
\hline Dinamara & 25 & 65 & ins & 3.3 & 6.7 & 26 & 13.0 & 6.1 & 10.0 & 5.6 & 10.0 \\
\hline Betgia & 2 & 4.1 & 10.8 & 3.1 & 4.9 & 26 & 7.7 & 80 & 30.0 & 3.1 & 7.0 \\
\hline Norvegen & 2 & 6.1 & 11.7 & 4.0 & 23 & 3.0 & 64 & 21 & 03 & 15 & II.0 \\
\hline Seacir & 2 & 6.1 & 14.0 & 29 & 33 & 24 & 43 & 21 & 0.3 & 1.0 & 10.0 \\
\hline Caneds & 25 & 45 & 128 & 4.1 & 43 & 26 & 9.1 & 7.0 & 5.0 & 2.1 & 8.0 \\
\hline Phive Brijos & 25 & 42 & 0.4 & 3.4 & 7.0 & 25 & 75 & 5.1 & 31.0 & 0.4 & 11.0 \\
\hline Esurias Unidas & 25 & 4.1 & 10.5 & 3.0 & 5.3 & 1.9 & 6.7 & 60 & 20 & 1.6 & 6.0 \\
\hline Alemenis & 4 & 3.0 & 55 & 3.4 & 5.6 & 33 & 8.6 & 3.6 & 9.0 & 2.6 & 3.0 \\
\hline Suizes & 4 & 3.2 & 6.1 & 2.7 & 8.6 & 25 & 10.0 & $N / A$ & N/A & 0.9 & 4.0 \\
\hline
\end{tabular}

Fuente: FMI, Summers y Heston, y OCDE. 
Cuadro A2 Apéndice

Independencia del Banco Central y resultados económicos en el período posterior a la crisis del petróleo

\begin{tabular}{|c|c|c|c|c|c|c|c|c|c|c|c|}
\hline Pis & $\begin{array}{l}\text { Indice promedio } \\
\text { de independencis } \\
\text { del Buncos Ceotral }\end{array}$ & $\begin{array}{l}\text { Inflación } \\
\text { promedio } \\
\text { 1973-88 }\end{array}$ & $\begin{array}{c}\text { Variación } \\
\text { en la inflación } \\
1973-88\end{array}$ & $\begin{array}{l}\text { Creciniento } \\
\text { promedio de] } \\
\text { PIB real 1973-87 }\end{array}$ & $\begin{array}{l}\text { Variccío en el } \\
\text { crecimienlo del } \\
\text { P1B real } 1973.87\end{array}$ & $\begin{array}{c}\text { Crecimiento } \\
\text { promedio del } \\
\text { PIB real per cipita } \\
1973-87\end{array}$ & $\begin{array}{l}\text { Vurimión en el } \\
\text { aecigiento del } \\
\text { PIB real per dpinde } \\
\text { 1973-87 }\end{array}$ & $\begin{array}{c}\text { Twe pronnedio } \\
\text { de deaneoki } \\
1973-88\end{array}$ & $\begin{array}{l}\text { Verición de } \\
\text { lates de } \\
\text { desempleo } \\
1973-88\end{array}$ & $\begin{array}{c}\text { Variwibo ded } \\
\text { interts ral } \\
197388\end{array}$ & $\begin{array}{r}\text { Verisción } \\
\text { del ioterts } \\
\text { real } 1973-60\end{array}$ \\
\hline Espais & 1.5 & 124 & 22.1 & 20 & 21 & 1.2 & 8.9 & N/A & N/A & N/A & N/A \\
\hline Nuevi Zelandia & 1 & 122 & 105 & 15 & 4.5 & 0.7 & 21.7 & N/A & N/A & -3 & 21.0 \\
\hline Australia & 20 & 9.5 & 7.3 & 28 & 3.2 & 1.4 & 7.2 & 66 & 3.0 & 1.6 & 21.0 \\
\hline Itadia & 1.75 & 125 & 29.6 & 24 & 4.9 & 29 & 7.7 & 8.4 & 4.0 & N/A & N/A \\
\hline Reino Unido & 2 & 67 & 23.5 & 1.6 & 4.1 & 20 & 7.8 & 8.8 & 15.0 & 0.9 & 27.0 \\
\hline Frencia & 2 & 8.2 & 126 & 21 & 1.3 & 15 & 4.1 & 7.0 & 6.0 & 21 & 10.0 \\
\hline Dinemara & 25 & 8.6 & 11.0 & 1.9 & 5.2 & 1.1 & 115 & 75 & 4.0 & 65 & 10.0 \\
\hline Belgia & 2 & 60 & 11.9 & 1.7 & 3.8 & 15 & 10.1 & 128 & 20.0 & 3.6 & 120 \\
\hline Nonegp & 2 & 8.2 & 5.6 & 3.9 & 3.3 & 3.0 & 6.3 & 22 & 0.3 & 24 & 14.0 \\
\hline Suecis & 2 & 8.3 & 7.6 & 1.0 & 21 & 1.5 & 5.6 & 23 & 0.3 & 1.7 & 17.0 \\
\hline Candi & 25 & 7.2 & 7.9 & 3.3 & 4.7 & 28 & 11.6 & 8.7 & 3.0 & 29 & 11.0 \\
\hline Pises Brios & 25 & 4.3 & 105 & 1.7 & 32 & 1.1 & 3.6 & 9.7 & 26.0 & 21 & 10.0 \\
\hline Japin & 25 & 45 & 17.1 & 3.7 & 2.8 & 26 & 05 & 23 & 0.2 & 24 & 7.0 \\
\hline Esindas Unidas & 25 & 6.4 & 11.1 & 24 & 65 & 1.6 & 9.8 & 7.2 & 1.0 & 21 & 11.0 \\
\hline Akeonanis & 4 & 3.4 & 4.0 & 1.8 & 33 & 1.8 & 6.9 & 6.2 & 6.0 & 3.0 & 3.0 \\
\hline Suiza & 4 & 3.1 & 43 & 1.0 & 8.1 & 1.4 & 11.0 & N/A & N/A & 1.6 & 3.0 \\
\hline
\end{tabular}

Fuente: FMI, Summers y Heston, y OCDE. 


\section{Referencias bibliograficas}

Alesina, Alberto, "Macroeconomics and Politics", en NBER Macroeconomics Annual, editado por Stanley Fischer, Cambridge, Mass.: MIT Press, pp. 17-52, 1988.

Alesina, Alberto, "Politics and Business Cycles in Industrial Democracies", Economic Policy, 8, 1989, pp. 58-98.

Alesina, Alberto y Grilli, Vittorio, "The European Central Bank: reshaping Monetary Policy in Europe", en Establishing a Central Bank: Issues in Europe and Lessons from the United Sarates, editado por Matthew Canzoneri, Vittorio Grilli y Paul Masson, Cambridge: Cambridge University Press y CEPR, 1992, pp. 49-77.

Bade, Robert y Parkin, Michael, "Central Bank Laws and Monetary Policy", inédito, 1982.

Barro, Robert y Gordon, David, "Rules, Discretion, and Reputation in a Model of Monetary Policy", Journal of Monetary Economics, 12, julio, 1983, pp. 101-22.

Barro, Robert y Sala-i-Martin, David, "World Real Intcrest Rates", en NBER Macroeconomics Annual, editado por Olivier Blanchard y Stanley Fischer, Cambridge, Mass.: MIT Press, 1990.

Black, Stanley, "Strategic Aspects of the Political Assignment Problem in Open Economics", en Political Economy of International and Domestic Monetary Reform, editado por Raymond Lombra y Willard Wille, lowa City: Iowa State University Press, 1982, pp. 130-52.

Cukicrman, Alex; Edwards, Sebastian y Tabellini, Guido, "Seignorage and Political Insability", American Economic Review, 82, junio, 1992, pp. 537-55.

Cukierman, Alex; Webb, Steven y Neyapli, Bilin, "The Measurement of Central Bank Independence and Its Effect on Policy Outcomes", incedito, 1991.

Grilli, Vittorio; Masciandaro, Donato y Tabellini, Guido, "Political and Monetary Institutions and Public Finance Policies in the Industrial Countries", Economic Policy, 13, oclubre, 1991, pp. 341-92.

Hibbs, Douglas, The American Political Economy, Cambridge, Mass.: Harvard University Press, 1987.

Kydland, Finn y Prescott, Edward, "Rules Rather than Discretion: The Inconsistency of Optimal Plans", Journol of Political Economy, 85, junio, 1977, pp. 473-90.

Nordhaus, William, "The Political Business Cycle", Review of Economic Studies, 42, abril, 1975, Pp. 169-90.

Rogoff, Kenneth, "The Optimal Degree of Commitment to an Intermediate Monetary Target", Quarterly Journal of Economic, 110, novicmbre, 1985, pp. 1169-90.

Rogoff, Kenneth y Sibert, Anne, "Equilibrium Political Business Cycles", Review of Economic Studies, 55, enero, 1988, pp. 1-16.

Romer, Christina y Romer, David, "Does Monetary Policy Malter? A Test in Ich Spirit of Friedman and Schwartz", en NBER Macroeconomics Annual, editado por Olivier Blanchard y Stanley Fischer, Cambridge, Mass.: MIT Pres, 1989, pp. 121-170.

Summers, Lawrence, "Comment on Postwar Developments in Business Cycle Theory: A Moderatcly Classical Perspective", Journal of Money, Credit, and Banking, 20, agoslo, 1988, pp. 472-76.

Summers, Lawrence y Wadwhani, Sushil, "The Determinants of the Cyclical Variability of Outpul", CLE Working Paper, 1989.

Willett, Thomas, ed., Political Business Cycles, Durham, N.C.: Duke University Press, 1988. 


\section{Notas}

1. Los autores están en deuda con Robert Barto por las útiles discusiones mantenidas; a dos referencias anónimas por sus comentarios y a Gerald Cohen y Jelfrey Manz por su asistencia en la investigación. Alesina agradece a la Fundación Sloan por su apoyo financiero.

Alberto Alesina es el profesor asociado de la cátedra Paul Sack de economía política en la Universidad de Harvard, y miembro del Centro de Investigaciones de Política Económica y de la Oficina Nacional de Investigaciones Económicas. Lawrence $\mathbf{H}$. Summers es prolesor de economía en la Universidad de Harvard y Asociado miembro de la Olicina Nacional de Investigaciones Económicas; Journal of Money, Credit, and Banking, Vol. 25, No. 2, mayo, 1993, derechos de autor 1993 de la Ohio State Universily Press.

2. Este volumen, además de los modelos de ciclos económicos políticos, consideran los modelos "burocráticos" de banca centrales, en los cuales los bancos centrales se ven como organismos burocráticos cuya mela es maximizar su influencia.

3. Para mayor información sobre la consırucción de este índice, véase Grilli, Masciandaro y Tabellini (1991).

4. Hablando de manera amplia, la diferencia entre los dos índices resulta más que todo del hecho que Grilli, Masciandaro y Tabellini (1991) colocan más peso que Bade y Parkin (1982) en las reglas relacionadas al financiamiento monetario de déficits gubernamentales.

5. El indice de Grilli, Masciandaro y Tabellini abarca los tres países escandinavos (Finlandia, Noruega y Suecia). Para dos de estos países se vililizó el índice de Bade y Parkin.

6. Este es el caso, por ejemplo, de Canadá, Italia y Nueva Zelandia. Los cambios en las leyes de los bancos centrales podrían ser endógenos, en el sentido de que los gobiernos pueden responder con reformas institucionales a periodos de alta inflación. Ver Cukierman, Webb y Neyapti (1991) para un análisis de este tema en una muestra grande de países.

7. Estos resultados son consisten con las comprobaciones similares obtenidas independientemente por Grilli, Masciandaro y Tabellini (1991), que utilizan una muestra y melodología diferentes.

8. Para un mayor análisis de las diferentes reglas y reformas intilucionales con referencia específica a la Reserva Federal, ver el último capílulo de Willlett (1988).

9. Para el análisis de varios otras delerminantes político institucionales de la inflación en diferentes países, ver, por ejemplo, Black (1982), varias contribuciones en Willett (1988), Grilli, Masciandaro y Tabellini (1991), Cukierman, Edward y Tabellini (1992). 